\title{
ON THE WAVE EQUATION ASSOCIATED TO THE HERMITE AND THE TWISTED LAPLACIAN
}

\author{
PIERO D'ANCONA, VITTORIA PIERFELICE, AND FULVIO RICCI
}

\begin{abstract}
The dispersive properties of the wave equation $u_{t t}+A u=0$ are considered, where $A$ is either the Hermite operator $-\Delta+|x|^{2}$ or the twisted Laplacian $-\left(\nabla_{x}-i y\right)^{2} / 2-\left(\nabla_{y}+i x\right)^{2} / 2$. In both cases we prove optimal $L^{1}-L^{\infty}$ dispersive estimates. More generally, we give some partial results concerning the flows $\exp \left(i t L^{\nu}\right)$ associated to fractional powers of the twisted Laplacian for $0<\nu<1$.
\end{abstract}

\section{INTRODUCTION}

The Hermite operator $H=-\Delta+|x|^{2}$ on $\mathbb{R}^{d}$ and the twisted Laplacian

$$
L=-\frac{1}{2} \sum_{j=1}^{d}\left[\left(\partial_{x_{j}}-i y_{j}\right)^{2}-\left(\partial_{y_{j}}+i x_{j}\right)^{2}\right] .
$$

on $\mathbb{R}^{2 d}$ have both a rich spectral theory and a basic geometric significance. Indeed, besides their interpretation as Schrödinger operators perturbed with electromagnetic fields, they are intimately connected with the sublaplacian on the Heisenberg group $\mathbb{H}^{d}$, which can be defined as the manifold $\mathbb{C}^{d} \times \mathbb{R}$ endowed with the Lie group product

$$
(z, t) \cdot(w, s)=\left(z+w, t+s+\frac{1}{2} \Im z \bar{w}\right) .
$$

In the coordinates $(z, t) \simeq(x, y, t)$, the sublaplacian $\mathcal{L}$ can be written as

$$
\mathcal{L}=-\Delta_{x, y}-\frac{1}{4}\left(|x|^{2}+|y|^{2}\right) \partial_{t}^{2}-\sum_{j=1}^{d}\left(x_{j} \partial_{y_{j}}-y_{j} \partial_{x_{j}}\right) \partial_{t} .
$$

Then the twisted Laplacian $L$ is related to $\mathcal{L}$ via

$$
L f(z)=e^{-i t} \mathcal{L}\left(e^{i t} f(z)\right)
$$

on functions $f(z)$ independent of the variable $t$. On the other hand, if we consider the operator valued group Fourier transform

$$
\hat{f}(\lambda)=\int f(z, t) \pi_{\lambda}(z, t) d z d t, \quad f \in L^{1}\left(\mathbb{H}^{d}\right)
$$

where $\pi_{\lambda}(z, t)$ is for each $(z, t) \in \mathbb{H}^{d}$ the operator on $L^{2}\left(\mathbb{R}^{d}\right)$ defined by

$$
\pi_{\lambda}(z, t) \phi(\xi)=e^{i \lambda(t+x \cdot \xi+x \cdot y / 2)} \phi(\xi+y),
$$

then the Hermite operator satisfies the identity

$$
\widehat{\mathcal{L f f}}(1)=\hat{f}(1) H .
$$

Here and in the following we refer to the standard literature on the subject (see e.g. [19])

Date: March 14, 2010.

2000 Mathematics Subject Classification. 35L05, 35Q40, 58J45, 11K70, 11L03 .

Key words and phrases. wave equation, Strichartz estimates, decay estimates, dispersive equations, Schrödinger equation, harmonic analysis, almost periodicity. 
From the spectral point of view, $H$ and $L$ are positive definite, selfadjoint operators on $L^{2}\left(\mathbb{R}^{d}\right)$ and $L^{2}\left(\mathbb{R}^{2 d}\right)$ with natural domains $D(H)=\left\{f \in L^{2}: H f \in L^{2}\right\}$, $D(L)=\left\{f \in L^{2}: L f \in L^{2}\right\}$. The spectrum is discrete and with strictly positive bottom; indeed,

$$
\sigma(H)=\sigma(L)=\{d+2 k: k=0,1,2, \ldots\} .
$$

The corresponding eigenspaces for $H$ are finite dimensional and are spanned by the basis of Hermite functions

$$
h_{\alpha}=(-1)^{|\alpha|} e^{\frac{|x|^{2}}{2}} \partial_{x}^{\alpha} e^{-|x|^{2}}, \quad H h_{\alpha}=(n+2|\alpha|) h_{\alpha} .
$$

On the other hand the eigenspaces are infinite dimensional for $L$, with basis

$$
f_{\alpha, \beta}=(-1)^{|\alpha|+|\beta|} e^{\frac{|z|^{2}}{4}} \partial_{z}^{\alpha} \partial_{\bar{z}}^{\beta} e^{\frac{|z|^{2}}{2}}, \quad L f_{\alpha, \beta}=(d+2|\alpha|) f_{\alpha, \beta}
$$

where we have used the complex notations

$$
z=x+i y, \quad \bar{z}=x-i y, \quad \partial_{z}=\frac{1}{2}\left(\nabla_{x}-i \nabla_{y}\right) \quad \partial_{\bar{z}}=\frac{1}{2}\left(\nabla_{x}+i \nabla_{y}\right) .
$$

In both cases we shall denote with $P_{k}$ the orthogonal projection on the eigespace of $d+2 k$. We recall that optimal $L^{p}$ estimates for the operators $P_{k}$ have been obtained in [9] and [8].

Moreover, in the case of the twisted Laplacian, the projections $P_{k}$ admit a simple representation using the normalized Laguerre functions

$$
\varphi_{k}(x, y)=e^{-\frac{|x|^{2}+|y|^{2}}{2}} L_{k}^{(d-1)}\left(\frac{|x|^{2}+|y|^{2}}{2}\right)
$$

(i.e. $\left\|\varphi_{k}\right\|_{L^{\infty}} \leq \varphi_{k}(0)=1$ ) and the twisted convolution operation

$$
\varphi \times f=\int_{\mathbb{R}^{2 d}} e^{i\left(x y^{\prime}-x^{\prime} y\right)} \varphi\left(x-x^{\prime}, y-y^{\prime}\right) f\left(x^{\prime}, y^{\prime}\right) d x^{\prime} d y^{\prime} .
$$

Notice that this normalization is not the standard one, but for simplicity we shall keep the standard notation $L_{k}^{d}$ for Laguerre polynomials. With these notations we have

$$
P_{k} f=\left(\begin{array}{c}
k+d-1 \\
d-1
\end{array}\right) \varphi_{k} \times f .
$$

The simplest and most important flows $e^{i t H} f, e^{i t L} f$ correspond to solutions of the Schrödinger equations

$$
i u_{t}+H u=0, \quad i u_{t}+L u=0
$$

with initial value $f$. In this case the dispersive properties can be read from the explicit representations

$$
e^{i t H} f(x)=\frac{C}{(\sin 2 t)^{d / 2}} \int e^{2 \frac{\left(|x|^{2}+\left|x^{\prime}\right|^{2}\right) \cos (2 t)-2 x x^{\prime}}{i \sin (2 t)}} f\left(x^{\prime}\right) d x^{\prime}
$$

which is a version of Mehler's formula, and

$$
e^{i t L} f(x, y)=\frac{C}{(\sin 2 t)^{d}} \int e^{2 \frac{\left|x-x^{\prime}\right|^{2}+\left|y-y^{\prime}\right|^{2}}{i \tan (2 t)}} e^{i\left(x y^{\prime}-x^{\prime} y\right)} f\left(x^{\prime}, y^{\prime}\right) d x^{\prime} d y^{\prime} .
$$

which can be derived by continuation of the corresponding heat kernel (see [11]). Then for small times we have the standard $L^{1}-L^{\infty}$ dispersive estimates

$$
\left|e^{i t H} f\right| \lesssim(\sin 2 t)^{-d / 2}\|f\|_{L^{1}}, \quad\left|e^{i t L} f\right| \lesssim(\sin 2 t)^{-d}\|f\|_{L^{1}}
$$

Notice that both flows are time-periodic, with first refocusing at $t=\pi / 2$. Thus no global in time dispersive properties can be expected; on the other hand, for small times $|t| \leq T(T<\pi / 2)$ the behaviour is identical to the free Schrödinger equation. As a consequence, the usual Ginibre-Velo-Keel-Tao argument applies and local Strichartz estimates hold for the full range of indices ([5], [7]). 
We also mention that a parametrix for the Schrödinger equations

$$
i u_{t}-(\nabla-i A(t, x))^{2} u+V(t, x) u=0
$$

perturbed with subquadratic electric potentials $V(t, x)$ and sublinear magnetic potentials $A(t, x)$, was constructed by K.Yajima ([21]). The above results can be obtained as special cases of this construction and thus hold in much greater generality.

The next natural flows to study are $e^{i t \sqrt{H}} f, e^{i t \sqrt{L}} f$, which describe solutions of the wave equations

$$
u_{t t}+H u=0, \quad u_{t t}+L u=0
$$

via the usual identities $(A=H$ or $L)$

$$
\cos (t \sqrt{A})=\frac{e^{i t \sqrt{A}}+e^{-i t \sqrt{A}}}{2}, \quad \frac{\sin (t \sqrt{A})}{\sqrt{A}}=\frac{e^{i t \sqrt{A}}-e^{-i t \sqrt{A}}}{2 i \sqrt{A}} .
$$

This case has received quite a deal of attention. Most works concern extensions of the well known sharp $L^{p}$ bound for the euclidean wave equation

$$
(1-\Delta)^{-\alpha / 2} e^{i t \sqrt{-\Delta}}: L^{p}\left(\mathbb{R}^{d}\right) \rightarrow L^{p}\left(\mathbb{R}^{d}\right)
$$

which is true provided

$$
\alpha \geq(d-1)\left|p^{-1}-2^{-1}\right|, \quad 1<p<\infty
$$

and also from the real Hardy space $H^{1}$ into $L^{1}$. The bound (1.8) was proved by Peral [15] and Miyachi [10] and extended in various directions, including general hyperbolic equations and FIOs [1], [16]; the wave equation associated to the Hermite operator [18]; and the case of the $\mathbb{H}^{d}$ sublaplacian [13], [12]. Tie and Wong [20] proved the finite speed of propagation in the case of the twisted Laplacian.

Our first goal here is to investigate the local dispersive properties of the flows $e^{i t \sqrt{H}} f$ and $e^{i t \sqrt{L}} f$. For the standard wave equation on $\mathbb{R}^{d}$ in the euclidean setting, the optimal dispersive estimate is most easily expressed in the frequency truncated form

$$
\left|\phi_{0}(\sqrt{-\Delta} / \lambda) e^{i t \sqrt{-\Delta}} f\right| \lesssim \lambda^{\frac{d+1}{2}} \cdot|t|^{-\frac{d-1}{2}}\|f\|_{L^{1}}
$$

where $\phi_{0}(r)$ is a fixed cutoff function with support in $[1 / 2,2]$, and a constant independent of the frequency $\lambda>0$. An equivalent global form of the same estimate requires the introduction of Besov spaces: assuming that $\phi_{0}$ generates a partition of unity $\phi_{j}(r)=\phi_{0}\left(2^{-j} r\right), j \in \mathbb{Z}$, with

$$
\sum_{j \in \mathbb{Z}} \phi_{j}(|x|)=1 \quad \text { for } x \neq 0 .
$$

and defining the homogeneous Besov norm $\dot{B}_{1,1}^{s}$ as

$$
\|f\|_{\dot{B}_{1,1}^{s}}=\sum_{j \in \mathbb{Z}} 2^{j s}\left\|\phi_{j}(\sqrt{-\Delta}) f\right\|_{L^{1}},
$$

we can sum estimates (1.9) over all dyadic frequencies obtaining the optimal dispersive estimate for the wave equation on $\mathbb{R}^{d}$

$$
\left|e^{i t \sqrt{-\Delta}} f\right| \lesssim|t|^{-\frac{d-1}{2}}\|f\|_{\dot{B}_{1,1}^{\frac{d+1}{2}}}
$$

(see e.g. [17])

In our first result we extend the above estimates to the wave flows for $H$ and $L$ : 
Theorem 1.1. Let $0<T<\pi / 2$. Let $\phi_{0}(r)$ be smooth and supported in $[1 / 2,2]$, and denote by $A$ either the Hermite operator $H$ on $\mathbb{R}^{d}$ or the twisted Laplacian $L$ on $\mathbb{R}^{2 d}$. Then the wave flow for $A$ satisfies the following dispersive estimate (where $n=d$ for $H$ and $n=2 d$ for $L)$ :

$$
\left|\phi_{0}\left(\lambda^{-1} \sqrt{A}\right) e^{i t \sqrt{A}} f\right| \lesssim \lambda^{\frac{n+1}{2}} \cdot|t|^{-\frac{n-1}{2}}\|f\|_{L^{1}}, \quad \lambda>0, \quad|t| \leq T .
$$

Remark 1.1. The proof of Theorem 1.1 is based on a subordination formula introduced by Müller and Seeger [12]; this method is quite solid and can be adapted to much more general situations, including e.g. potential perturbations with quadratic growth for the electric part and linear growth for the magnetic part. In particular, we can prove a local in time, optimal dispersive estimate (i.e., identical to the case of the free wave equation) for any wave equation perturbed with a constant magnetic field

$$
u_{t t}-\sum_{j}\left(\partial_{j}-i a_{j} x_{j}\right)^{2} u=0
$$

We omit the straightforward details.

Remark 1.2. Summing (1.12) over dyadic frequencies, we obtain an estimate like (1.11) with a distorted Besov norm, based on the operators $H$ or $L$ instead of $-\Delta$ :

$$
\|f\|_{B_{1,1}^{s}(A)}=\sum_{j \in \mathbb{N}} 2^{j s}\left\|\phi_{j}(\sqrt{A}) f\right\|_{L^{1}}, \quad A=H \text { or } L .
$$

Notice that here the possible frequencies are bounded from below since the spectrum is strictly positive and discrete, hence we can drop from the sum the terms with dyadic $2^{j}$ for $j \leq 0$ (assuming in addition that $\phi_{0}(r)=1$ on $[1,3 / 2]$ ). Thus in particular the distorted homogeneous Besov norm coincides with the non-homogeneous one $B_{1,1}^{s}(A)$. For a thorough study of the spaces $B_{p, q}^{s}(H), B_{p, q}^{s}(L)$ we refer to [14], [3], [2]. A natural question is to compare such distorted norms with the standard ones (1.10); we shall not pursue this problem here.

As mentioned above, the proof of Theorem 1.1 is based on a reduction to the corresponding Schrödinger flow. This gives a very precise result for small times, but has an important drawback too. Indeed, the Schrödinger flow is time periodic, and in particular the $L^{1}-L^{\infty}$ dispersive estimate fails at $t=\pi / 2$, the best bound being of course the Sobolev embedding

$$
\left\|\phi_{0}\left(\lambda^{-1} \sqrt{L}\right) \exp (i \pi L / 2) f\right\|_{L^{\infty}} \equiv\left\|\phi_{0}\left(\lambda^{-1} \sqrt{L}\right) f\right\|_{L^{\infty}} \lesssim \lambda^{2 d}\|f\|_{L^{1}}
$$

(more properly, this should be called a Berstein type inequality; see Lemma 2.1). As a consequence our method breaks down at $t=\pi / 2$ and a condition like $|t| \leq$ $T<\pi / 2$ is necessary in the statement.

On the other hand, it is clear that the wave flow is not periodic but quasiperiodic in a suitable sense, which can be made precise at least for initial data containing a finite number of frequencies. Thus we expect that no refocusing effect should occur for $t \neq 0$. In the following we restrict our attention to the case of the twisted Laplacian $L$, and we consider a general fractional flow

$$
\exp \left(i t L^{\nu}\right) f, \quad 0<\nu<1,
$$

which represents the solution to the Cauchy problem

$$
i u_{t}+L^{\nu} u=0, \quad u(0, x)=f(x) .
$$

For $\nu=1 / 2$ we are back to the wave flow. The main advantage here is that the flow can be represented quite explicitly via an exponential sum like

$$
e^{i t L^{\nu}} L^{-\alpha} f=\sum_{k \geq 0} \frac{e^{i t(d+2 k)^{\nu}}}{(d+2 k)^{\alpha}}\left(\begin{array}{c}
k+d-1 \\
d-1
\end{array}\right) \varphi_{k} \times f
$$


with the notations introduced above. Our second result is the following:

Theorem 1.2. Let $d \geq 2$. For all $0<\nu<1, d>\alpha>d-\min \{\nu, 1-\nu\}$, we have the estimate

$$
\left\|e^{i t L^{\nu}} L^{-\alpha} f\right\|_{L^{\infty}\left(\mathbb{R}^{2 d}\right)} \leq C\left(|t|^{\frac{\alpha-d}{\nu}}+|t|\right)\|f\|_{L^{1}\left(\mathbb{R}^{2 d}\right)} \quad \text { for } \quad t \neq 0 .
$$

In order to better appreciate the result, we specialize it to the case $\nu=1 / 2$. If we apply estimate (1.14) to a solution truncated at frequency $\lambda$, using again the Bernstein type inequalities of Lemma 2.1, we obtain

$$
\left|\phi_{0}\left(\lambda^{-1} \sqrt{L}\right) e^{i t \sqrt{L}} f\right| \lesssim \lambda^{2 \alpha} \cdot\left(|t|^{2(\alpha-d)}+|t|\right)\|f\|_{L^{1}}, \quad d>\alpha>d-1 / 2 .
$$

Compare this with the dispersive estimate of Theorem 1.1, which can be written $(|t| \leq T<\pi / 2, C=C(T))$

$$
\left|\phi_{0}\left(\lambda^{-1} \sqrt{L}\right) e^{i t \sqrt{L}} f\right| \lesssim \lambda^{2 \alpha} \cdot|t|^{2(\alpha-d)}\|f\|_{L^{1}}, \quad \alpha=\frac{d}{2}+\frac{1}{4}
$$

and with the Sobolev embedding (any $t$ )

$$
\left|\phi_{0}\left(\lambda^{-1} \sqrt{L}\right) e^{i t \sqrt{L}} f\right| \lesssim \lambda^{2 \alpha} \cdot|t|^{2(\alpha-d)}\|f\|_{L^{1}}, \quad \alpha=d .
$$

Thus for small times (1.15) is an interpolation between the optimal dispersive estimate and Sobolev embedding. However, (1.15) holds for all $t \neq 0$, and gives an improvement over Sobolev embedding which can be regarded as a measure of the non-refocusing of the wave flow for large times.

\section{Proof of Theorem 1.1}

A useful tool to handle frequency truncations are $L^{p}$ Bernstein inequalities, which can be extended to the present setting:

Lemma 2.1 (Bernstein type inequalities). Let $\phi_{0}(x)$ a radial cutoff supported in $1 / 2 \leq|x| \leq 2$. Then the following estimate holds, for any $f \in L^{p}\left(\mathbb{R}^{d}\right), \alpha \in \mathbb{R}$ and $\lambda>0$ :

$$
\left\|\phi_{0}\left(\lambda^{-1} \sqrt{H}\right) H^{\alpha} f\right\|_{L^{q}} \lesssim \lambda^{2 \alpha+\frac{d}{p}-\frac{d}{q}}\|f\|_{L^{p}}, \quad 1 \leq p \leq q \leq \infty .
$$

An analogous estimate holds for the twisted Laplacian $L$ : for any $f \in L^{p}\left(\mathbb{R}^{2 d}\right)$, $\alpha \in \mathbb{R}$ and $\lambda>0$ :

$$
\left\|\phi_{0}\left(\lambda^{-1} \sqrt{L}\right) L^{\alpha} f\right\|_{L^{q}} \lesssim \lambda^{2 \alpha+\frac{2 d}{p}-\frac{2 d}{q}}\|f\|_{L^{p}}, \quad 1 \leq p \leq q \leq \infty .
$$

Proof. This kind of estimate is quite standard; we sketch a proof for the sake of completeness. It is well known ([3], [14]) that the integral kernel of $\phi_{0}\left(\lambda^{-1} \sqrt{H}\right)$ satisfies for all $N \geq 0$

$$
\left|\phi_{0}\left(\lambda^{-1} \sqrt{H}\right)(x, y)\right| \leq C_{N} \frac{\lambda^{n}}{(1+\lambda|x-y|)^{N}}
$$

with a constant independent of $\lambda$; a similar estimate is satisfied by $\phi_{0}\left(\lambda^{-1} \sqrt{L}\right)$. Actually this holds for any operator whose heat kernel satisfies a gaussian bound

$$
\left|e^{-t H}(x, y)\right| \leq c_{1} t^{-d / 2} e^{-c|x-y|^{2} / t}, \quad c>0
$$

as proved in [14] (Proposition 5.1). By scaling, this extends to the slightly more general estimate

$$
\left|H^{\alpha} \phi_{0}\left(\lambda^{-1} \sqrt{H}\right)(x, y)\right| \leq C_{N} \frac{\lambda^{d+2 \alpha}}{(1+\lambda|x-y|)^{N}} .
$$

Then by Young's inequality we deduce immediately (2.1), and in a similar way we obtain (2.2). 
We shall now prove Theorem 1.1 for the operator $L$; the proof for $H$ is identical. We distinguish two cases: the high frequency case $\lambda t \geq 1$, and the low frequency case $\lambda t \leq 1$.

For the high frequency case, we shall use the following subordination formula from $[12]$ :

Proposition 2.2. Let $\phi_{0}(s) \in C_{c}^{\infty}(\mathbb{R})$ be supported in $[1 / 2,2]$. There exist a symbol $a_{0} \in C^{\infty}(\mathbb{R} \times \mathbb{R})$ with $\operatorname{supp} a(\cdot, \sigma) \subseteq[1 / 16,4]$ for every $\sigma \in \mathbb{R}$, satisfying

$$
\left|\partial_{s}^{j} \partial_{\sigma}^{k} a_{0}(s, \sigma)\right| \leq C_{j, k}(1+|s|)^{-k} \quad \forall j, k \geq 0
$$

and a Schwartz class function $\rho(s, \sigma) \in \mathcal{S}\left(\mathbb{R}^{2}\right)$ such that

$$
\phi_{0}\left(\frac{\sqrt{x}}{\lambda}\right) e^{i t \sqrt{x}}=\rho\left(\frac{t x}{\lambda}, \lambda t\right)+\phi_{0}\left(\frac{\sqrt{x}}{\lambda}\right) \sqrt{\lambda t} \int a_{0}(s, \lambda t) e^{i \frac{\lambda t}{4 s}} e^{i \frac{t s}{\lambda} x} d s,
$$

for every $\lambda, t, x \geq 0$ with $\lambda t \geq 1$.

For the convenience of the reader, we give an independent proof of the formula in the appendix at the end of the paper.

By spectral calculus for the nonnegative selfadjoint operator $L$ on $L^{2}\left(\mathbb{R}^{2 d}\right)$, using Proposition 2.2 we can represent the operator $\phi_{0}\left(\lambda^{-1} \sqrt{L}\right) e^{i t \sqrt{L}}$ as a sum

$$
\phi_{0}\left(\lambda^{-1} \sqrt{L}\right) e^{i t \sqrt{L}}=A_{\lambda}+B_{\lambda}
$$

where

$$
A_{\lambda}=\rho\left(\lambda^{-1} t L, \lambda t\right)
$$

and

$$
B_{\lambda}=\phi_{0}\left(\lambda^{-1} \sqrt{L}\right) \sqrt{\lambda t} \int a_{0}(s, \lambda t) e^{i \frac{\lambda t}{4 s}} e^{i \frac{t s}{\lambda} L} d s .
$$

For all real $N \geq 0$ we have

$$
\left|\rho\left(\lambda^{-1} t x, \lambda t\right)\right| \leq C_{N}\langle\lambda t\rangle^{-N} \quad \forall \lambda, x, t>0
$$

since $\rho$ is Schwartz class. Notice from expression (2.3) that also $\rho$ is supported in $\lambda / 2 \leq \sqrt{x} \leq 2 \lambda$, hence we have

$$
A_{\lambda} \equiv \psi_{0}\left(\frac{\sqrt{L}}{\lambda}\right) A_{\lambda}
$$

where $\psi_{0}$ is supported in $[1 / 4,4]$ and is equal to 1 on the support of $\phi_{0}$. Then using the Bernstein inequalities (2.1) we can write

$$
\begin{aligned}
\left\|A_{\lambda} f\right\|_{L^{\infty}} & \lesssim \lambda^{d}\left\|A_{\lambda} f\right\|_{L^{2}} \lesssim \lambda^{d}\langle\lambda t\rangle^{-N}\left\|\psi_{0}\left(\lambda^{-1} \sqrt{L}\right) f\right\|_{L^{2}} \\
& \lesssim \lambda^{1 / 2} t^{-d+1 / 2}\left\|\psi_{0}\left(\lambda^{-1} \sqrt{L}\right) f\right\|_{L^{2}}
\end{aligned}
$$

where we have chosen $N=d-1 / 2$. Hence, again by $(2.1)$,

$$
\left\|A_{\lambda} f\right\|_{L^{\infty}} \lesssim \lambda^{d+1 / 2} t^{-d+1 / 2}\|f\|_{L^{1}} .
$$

We now estimate $B_{\lambda}$. As recalled in the Introduction, the Schrödinger flow associated to $L$ satisfies the dispersive estimate

$$
\left|e^{i t L} f\right| \lesssim t^{-d}\|f\|_{L^{1}}
$$

for $0<t<T<\pi / 2$. This gives immediately

$$
\begin{aligned}
\left\|B_{\lambda} f\right\|_{L^{\infty}} & \lesssim \sqrt{\lambda t} \int\left|a_{0}(s, \lambda t)\right|\left(t s \lambda^{-1}\right)^{-d}\|f\|_{L^{1}} d s \\
& =\lambda^{d+1 / 2} t^{-d+1 / 2}\|f\|_{L^{1}} \cdot \int\left|a_{0}(s, \lambda t)\right| s^{-d} d s
\end{aligned}
$$


Summing up, we have proved the estimate

$$
\left\|\phi_{0}\left(\lambda^{-1} \sqrt{L}\right) e^{i t \sqrt{L}} f\right\|_{L^{\infty}} \lesssim \lambda^{d+1 / 2} t^{-d+1 / 2}\|f\|_{L^{1}}
$$

for all $\lambda, t>0$ such that $\lambda t \geq 1$.

It remains to estimate the case $\lambda t \leq 1$. This is especially easy using (2.1) since we can write

$$
\left\|\psi_{0}\left(\lambda^{-1} \sqrt{L}\right) e^{i t \sqrt{L}} f\right\|_{L^{\infty}} \lesssim \lambda^{2 d}\left\|\psi_{0}\left(\lambda^{-1} \sqrt{L}\right) f\right\|_{L^{1}}
$$

but now $\lambda \leq t^{-1}$ and hence

$$
\lambda^{2 d}=\lambda^{d+1 / 2} \lambda^{d-1 / 2} \leq \lambda^{d+1 / 2} t^{-d+1 / 2}
$$

which gives the same estimate as before.

\section{A Dirichlet series estimate}

The proof of Theorem 1.2 is based on a direct estimate of the exponential sum (1.13). The crucial tool is the following estimate (3.2) for a series of Dirichlet type. This kind of exponential sum appeared earlier in the literature (e.g. they are briefly mentioned in [6]) but only questions of convergence have been addressed; the pointwise estimate (3.2) seems new.

Lemma 3.1. Let $M, A, B, \nu$ be nonnegative real numbers with

$$
B>\min \{\nu, 1-\nu\} \text {. }
$$

Then the following estimate holds for $t>0, \epsilon \geq 0$ :

$$
\left|\sum_{k>M} \frac{e^{(i t-\epsilon)(k+A)^{\nu}}}{(k+A)^{B}}\right| \lesssim \frac{1}{(M+A)^{B}}\left[\frac{1}{t(M+A)^{\nu-1}}+1+t(M+A)^{\nu}\right]
$$

with a constant independent of $M, A, t, \epsilon$.

Proof. Denote by $k_{0}$ the minimal integer $>M$ and write the sum to be estimated as follows:

$$
\sum_{k>M} \frac{e^{(i t-\epsilon)(k+A)^{\nu}}}{(k+A)^{B}}=I-I I-I I I
$$

where

$$
\begin{gathered}
I=\int_{M}^{\infty} \frac{e^{(i t-\epsilon)(x+A)^{\nu}}}{(x+A)^{B}} d x, \quad I I=\int_{k_{0}}^{M} \frac{e^{(i t-\epsilon)(x+A)^{\nu}}}{(x+A)^{B}} d x, \\
I I I=\sum_{k>M}\left(\int_{k}^{k+1} \frac{e^{(i t-\epsilon)(x+A)^{\nu}}}{(x+A)^{B}} d x-\frac{e^{(i t-\epsilon)(k+A)^{\nu}}}{(k+A)^{B}}\right) .
\end{gathered}
$$

In order to estimate the first term $I$, we perform the change of variable $(x+A)^{\nu}=$ $s t^{-1}$ to obtain

$$
|I|=\nu^{-1} t^{\frac{B-1}{\nu}}\left|\int_{t(M+A)^{\nu}}^{+\infty} e^{i s} e^{-\epsilon s t^{-1}} s^{\frac{1-B}{\nu}-1} d s\right| \leq C t^{-1}(M+A)^{1-B-\nu}
$$

where we used the fact that, for any $\sigma>0$,

$$
\left|\int_{Z}^{+\infty} e^{i s-K s} s^{-\sigma} d s\right| \leq C Z^{-\sigma}
$$

uniformly in $Z>0$ and $K \geq 0$ (write $e^{i s-K s}=(i-K)^{-1} \partial_{s}\left(e^{i s-K s}\right)$ and integrate by parts). This gives the constraint $B>1-\nu$.

For the second term $I I$, since $0 \leq k_{0}-M \leq 1$, we have simply

$$
|I I| \leq \int_{M}^{k_{0}} \frac{d x}{(x+A)^{B}} \leq \frac{1}{(M+A)^{B}} .
$$


Finally, we rewrite the last term as follows

$$
\begin{aligned}
I I I & =\sum_{k>M} \int_{k}^{k+1} d x \int_{k}^{x} d y \frac{d}{d y}\left(\frac{e^{(i t-\epsilon)(y+A)^{\nu}}}{(y+A)^{B}}\right) \\
& =\sum_{k>M} \int_{k}^{k+1}(k+1-y) \frac{d}{d y}\left(\frac{e^{(i t-\epsilon)(y+A)^{\nu}}}{(y+A)^{B}}\right) d y,
\end{aligned}
$$

and noticing that $|k+1-y| \leq 1$ we have

$$
|I I I| \leq \sum_{k>M} \int_{k}^{k+1} \frac{\left|(i t-\epsilon) \nu(y+A)^{\nu}-B\right|}{(y+A)^{B+1}} e^{-\epsilon(y+A)^{\nu}} d y
$$

which gives

$$
|I I I| \leq \int_{M}^{\infty} \frac{t \nu}{(y+A)^{B+1-\nu}} d y+\int_{M}^{\infty} \frac{B}{(y+A)^{B+1}} d y+\int_{M}^{\infty} \frac{\epsilon(y+A)^{\nu} e^{-\epsilon(y+A)^{\nu}}}{(y+A)^{B+1}} d y .
$$

Notice that $r e^{-r} \leq 1$, hence we can write

$$
|I I I| \leq \frac{C t}{(M+A)^{B-\nu}}+\frac{C}{(M+A)^{B}}
$$

provided $B-\nu>0$ and $B>0$. This concludes the proof of the lemma.

We can now prove Theorem 1.2. Given the series expansion

$$
e^{i t L^{\nu}} L^{-\alpha} f=\sum_{k \geq 0} \frac{e^{i t(d+2 k)^{\nu}}}{(d+2 k)^{\alpha}}\left(\begin{array}{c}
k+d-1 \\
d-1
\end{array}\right) \varphi_{k} \times f
$$

we have to prove the estimate for all $t \neq 0$

$$
\left\|e^{i t L^{\nu}} L^{-\alpha} f\right\|_{L^{\infty}\left(\mathbb{R}^{2 d}\right)} \lesssim\left(|t|^{\frac{\alpha-d}{\nu}}+|t|\right)\|f\|_{L^{1}\left(\mathbb{R}^{2 d}\right)},
$$

provided $d \geq 2, d>\alpha>d-\min \{\nu, 1-\nu\}, 0<\nu<1$, with a constant depending only on $\alpha, \nu, d$.

We can assume $t>0$. By the Young inequality for twisted convolutions we see that the estimate is equivalent to

$$
\left|\sum_{k \geq 0} \frac{e^{i t(d+2 k)^{\nu}}}{(d+2 k)^{\alpha}}\left(\begin{array}{c}
k+d-1 \\
d-1
\end{array}\right) \varphi_{k}\right| \lesssim t^{\frac{\alpha-d}{\nu}}+t
$$

for $t>0$. We split the sum in two parts $I+I I$, with

$$
I=\sum_{2 k+d \leq t^{-1 / \nu}} \text { and } I I=\sum_{2 k+d>t^{-1 / \nu}} .
$$

In part $I$ the oscillations of the exponential are ineffective; recalling that $\left|\varphi_{k}\right| \leq 1$, it is sufficient to write

$$
|I| \leq \sum_{2 k+d \leq t^{-1 / \nu}} \frac{1}{(d+2 k)^{\alpha}}\left(\begin{array}{c}
k+d-1 \\
d-1
\end{array}\right) .
$$

Noticing that

$$
\left(\begin{array}{c}
k+d-1 \\
d-1
\end{array}\right)=\sum_{j=0}^{d-1} c_{j}(d+2 k)^{j}
$$

for suitable integers $c_{j}$, we are reduced to estimate the elementary sums

$$
\sum_{2 k+d \leq t^{-1 / \nu}} \frac{1}{(d+2 k)^{\alpha-j}}, \quad 0 \leq j \leq d-1
$$


which are all dominated by the worst case $j=d-1$

$$
\sum_{2 k+d \leq t^{-1 / \nu}} \frac{1}{(d+2 k)^{\alpha-d+1}}
$$

Under the condition $\alpha<d$, this is a divergent series and its partials can be easily estimated; we obtain

$$
|I| \lesssim t^{\frac{\alpha-d}{\nu}}
$$

Notice that for large values of $t$ the sum is empty.

Consider now the terms in $I I$. We shall use the following summation by parts formula to rearrange the series:

$$
\sum_{n=n_{0}}^{N} a_{n} b_{n}=\sum_{n=n_{0}+1}^{N}\left(a_{n}-a_{n-1}\right) \sum_{k=n}^{N} b_{k}+a_{n_{0}} \sum_{k=n_{0}}^{N} b_{k}
$$

Thus, denoting by $k_{0}$ the minimal integer $k$ such that $2 k+d>t^{-1 / \nu}$ and choosing (for a fixed $z$ )

$$
a_{k}=\left(\begin{array}{c}
k+d-1 \\
d-1
\end{array}\right) \varphi_{k}(z) \text { and } \quad b_{k}=\frac{e^{i t(d+2 k)^{\nu}}}{(d+2 k)^{\alpha}}
$$

we obtain

$$
I I=I I_{1}+I I_{2}
$$

with

$$
I I_{1}=\sum_{2 k+d>t^{-1 / \nu}+2}\left[\left(\begin{array}{c}
k+d-1 \\
d-1
\end{array}\right) \varphi_{k}-\left(\begin{array}{c}
k+d-2 \\
d-1
\end{array}\right) \varphi_{k-1}\right] \sum_{j=k}^{\infty} \frac{e^{i t(d+2 j)^{\nu}}}{(d+2 j)^{\alpha}}
$$

and

$$
I I_{2}=\left(\begin{array}{c}
k_{0}+d-1 \\
d-1
\end{array}\right) \varphi_{k_{0}} \sum_{2 k+d>t^{-1 / \nu}} \frac{e^{i t(d+2 k)^{\nu}}}{(d+2 k)^{\alpha}} .
$$

We estimate the second part $I I_{2}$ as follows

$$
\left|I I_{2}\right| \lesssim\left(d+2 k_{0}\right)^{d-1}\left|\sum_{2 k+d>t^{-1 / \nu}} \frac{e^{i t(d+2 k)^{\nu}}}{(d+2 k)^{\alpha}}\right|
$$

and applying directly Lemma 3.1 we obtain

$$
\left|I I_{2}\right| \lesssim\left(d+2 k_{0}\right)^{d-\alpha}\left[\frac{1}{t\left(d+2 k_{0}\right)^{\nu-1}}+1+t\left(d+2 k_{0}\right)^{\nu}\right] .
$$

provided $\alpha>0, \alpha>\nu, \alpha>1-\nu$. Since by definition $d+2 k_{0} \simeq t^{-1 / \nu}+1$, by separating the cases $t \leq 1$ and $t \geq 1$ we conclude easily that

$$
\left|I I_{2}\right| \lesssim t^{\frac{\alpha-d}{\nu}}+t
$$

We then rewrite $I I_{1}$ using the recurrence formula for Laguerre polynomials (see e.g. Section 10.12 of [4])

$$
\left(\begin{array}{c}
k+d-1 \\
d-1
\end{array}\right) L_{k}^{(d-1)}-\left(\begin{array}{c}
k+d-2 \\
d-2
\end{array}\right) L_{k-1}^{(d-1)}=\left(\begin{array}{c}
k+d-2 \\
d-2
\end{array}\right) L_{k}^{(d-2)}
$$

recall that in our notation $\varphi_{k}$ differs from $L_{k}^{(d-1)}$ only by a gaussian factor. This gives

$$
\left|\left(\begin{array}{c}
k+d-1 \\
d-1
\end{array}\right) \varphi_{k}-\left(\begin{array}{c}
k+d-2 \\
d-2
\end{array}\right) \varphi_{k-1}\right| \lesssim(d+2 k)^{d-2}
$$


We plug the inequality into $I I_{1}$ to obtain

$$
\left|I I_{1}\right| \lesssim \sum_{2 k+d>t^{-1 / \nu}}(d+2 k)^{d-2}\left|\sum_{j=k}^{\infty} \frac{e^{i t(d+2 j)^{\nu}}}{(d+2 j)^{\alpha}}\right|
$$

Again by Lemma 3.1 we derive that

$$
\left|I I_{1}\right| \lesssim \sum_{2 k+d>t^{-1 / \nu}}(d+2 k)^{d-2} \frac{1}{(d+2 k)^{\alpha}}\left[\frac{1}{t(d+2 k)^{\nu-1}}+1+t(d+2 k)^{\nu}\right] .
$$

If $d-\alpha-\nu<0, d-1-\alpha<0, d+\nu-\alpha-1<0$, we can sum the three convergent series and we obtain as above

$$
\left|I I_{1}\right| \lesssim\left(d+2 k_{0}\right)^{d-\alpha}\left[\frac{1}{t\left(d+2 k_{0}\right)^{\nu-1}}+1+t\left(d+2 k_{0}\right)^{\nu}\right]
$$

and by the same argument we deduce

$$
\left|I I_{1}\right| \lesssim t^{\frac{\alpha-d}{\nu}}+t
$$

which concludes the proof of the Theorem.

\section{Appendix A. Proof of Proposition 2.2}

We begin by estimating an integral of the form

$$
I(p, t)=\int_{0}^{\infty} e^{i t / 4 s} e^{i p s} s^{-3 / 2} d s, \quad p, t>0 .
$$

The integral is not absolutely convergent for $s \sim 0$; we shall define it as the limit for $\epsilon, \delta \rightarrow 0^{+}$of

$$
I_{\epsilon, \delta}(p, t)=\int_{0}^{\infty} e^{i t / 4 s-\epsilon / 4 s} e^{i p s-\delta s} s^{-3 / 2} d s .
$$

Let $\psi_{j}(s), j=1,2,3$ be three functions in $C^{\infty}(\mathbb{R})$ with

$$
\sum_{j=1}^{3} \psi_{j}=1, \quad \operatorname{supp} \psi_{1} \subseteq[3 / 2, \infty), \quad \operatorname{supp} \psi_{2} \subseteq(-\infty, 1 / 8], \quad \operatorname{supp} \psi_{3} \subseteq[1 / 16,2]
$$

and write for $j=1,2,3$

$$
I^{j}(p, t)=\int_{0}^{\infty} e^{i t / 4 s} e^{i p s} s^{-3 / 2} \psi_{j}(s) d s
$$

so that $I=I^{1}+I^{2}+I^{3}$. We shall denote with $I_{\epsilon, \delta}=I_{\epsilon, \delta}^{1}+I_{\epsilon, \delta}^{2}+I_{\epsilon, \delta}^{3}$ the corresponding decomposition of the regularized integral $I_{\epsilon, \delta}$.

Consider the first piece $I^{1}$. The phase in the oscillating factor is $p s+t / 4 s$ with derivative $p-t / 4 s^{2}$; since the cutoff restricts $s$ to $[3 / 2,+\infty[$, we have

$$
p-\frac{t}{4 s^{2}} \geq \frac{p+t}{8} \quad \text { for } \quad p \geq \frac{t}{4} \geq 0
$$

and this gives

$$
p \geq \frac{t}{4}>0 \Longrightarrow\left|I^{1}(p, t)\right| \leq C_{N}(p+t)^{-N} \quad \text { for all } N
$$

by a non-stationary phase argument. Moreover after an integration by parts we can write

$$
I^{1}=i \int_{0}^{\infty} e^{i t / 4 s} e^{i p s} \frac{d}{d s}\left(\left(p-1 / 4 s^{2}\right)^{-1} s^{-3 / 2} \psi_{1}(s)\right) d s .
$$

Expanding the derivative shows that the new integral has a better convergence since the various terms decay at least as $s^{-5 / 2}$. Thus we can differentiate $I^{1}$ with 
respect to $p, t$ and repeat the non-stationary phase argument. The same holds for higher order derivatives. We conclude that

$$
p \geq \frac{t}{4}>0 \Longrightarrow\left|\partial_{p}^{\alpha} \partial_{t}^{\beta} I^{1}(p, t)\right| \leq C_{N, \alpha, \beta}(p+t)^{-N} \quad \text { for all } N, \alpha, \beta .
$$

It is elementary to check that $I_{\epsilon, \delta}^{1}$ satisfies the same estimate with the same constants independent of $\epsilon, \delta$, and that $I_{\epsilon, \delta}^{1} \rightarrow I^{1}$ as $\epsilon, \delta \rightarrow 0^{+}$.

Consider now the singular piece $I^{2}(p, t)$; if we formally integrate by parts as above we can write

$$
I^{2}=-i \int_{0}^{\infty} e^{i t / 4 s} e^{i p s} \frac{d}{d s}\left(\frac{4 s^{1 / 2} \psi_{2}(s)}{\left(t-4 s^{2} p\right)}\right) d s .
$$

which is now a convergent integral. We can make this argument rigorous by performing a similar computation on $I_{\epsilon, \delta}^{2}$ and then taking the limit as $\epsilon, \delta \rightarrow 0^{+}$. This proves both that $I_{\epsilon, \delta}^{2}$ converges and that its limit $I^{2}$ can be equivalently written in the form (A.4). Noticing that

$$
t \geq \frac{p}{4}>0 \Longrightarrow t-4 s^{2} p \geq t-\frac{p}{16} \geq \frac{t}{2} \geq \frac{p+t}{32}
$$

since $s<\frac{1}{8}$ in the integral, and proceeding as above, we obtain now

$$
t \geq \frac{p}{4}>0 \Longrightarrow\left|\partial_{p}^{\alpha} \partial_{t}^{\beta} I_{2}(p, t)\right| \leq C_{N, \alpha, \beta}(p+t)^{-N} \quad \text { for all } N, \alpha, \beta .
$$

The last term $I^{3}$ can be written as

$$
I^{3}(p, t)=\int_{0}^{\infty} e^{i t / 4 s} e^{i p s} a(s) d s
$$

where $a(s)=s^{-3 / 2} \psi_{3}(s)$ is supported in $[-1 / 16,2]$.

Summing up we have proved that $I(p, t)$ is well defined and can be decomposed as follows

(A.7) $I(p, t)=\sigma(p, t)+\int_{0}^{\infty} e^{i t / 4 s} e^{i p s} a(s) d s, \quad a \in C_{0}^{\infty}, \quad \operatorname{supp} a \subseteq[1 / 16,2]$

where $\sigma=I^{1}+I^{2}$ satisfies

$$
\left|\partial_{p}^{\alpha} \partial_{t}^{\beta} \sigma(p, t)\right| \leq C_{N, \alpha, \beta}(p+t)^{-N} \quad \text { for } \quad \frac{1}{4} \leq \frac{p}{t} \leq 4
$$

We recall now the classical subordination formula

$$
e^{-y \sqrt{x}}=\frac{y}{2 \sqrt{\pi}} \int_{0}^{\infty} e^{-y^{2} / 4 s} e^{-s x} s^{-3 / 2} d s, \quad x, y>0 .
$$

Changing $s$ to sy and extending in the complex plane to $y=\epsilon-i t, \epsilon>0$ gives the identity

$$
e^{i t \sqrt{x}} e^{-\epsilon \sqrt{x}}=\frac{1}{2 \sqrt{\pi}}(\epsilon-i t)^{1 / 2} \int_{0}^{\infty} e^{i t / 4 s} e^{i t x s} e^{-\epsilon / 4 s} e^{-\epsilon x s} d s
$$

with the choice

$$
(\epsilon-i t)^{1 / 2}=\frac{t}{2 \sqrt{\gamma}}-i \sqrt{\gamma}, \quad \gamma=\frac{\left(t^{2}+\epsilon^{2}\right)^{1 / 2}-\epsilon}{2} .
$$

This can be expressed using the integral $I_{\epsilon, \delta}$ defined above as

$$
e^{i t \sqrt{x}} e^{-\epsilon \sqrt{x}}=\frac{1}{2 \sqrt{\pi}}(\epsilon-i t)^{1 / 2} I_{\epsilon, x \epsilon}(x t, t) .
$$

Taking the limit $\epsilon \rightarrow 0^{+}$we obtain the formula

$$
2 \pi^{1 / 2} e^{i t \sqrt{x}}=\sqrt{t} \cdot I(x t, t)=\sqrt{t} \cdot \sigma(x t, t)+\sqrt{t} \int_{0}^{\infty} e^{i t / 4 s} e^{i p s} a(s) d s
$$


with $\sigma, a(s)$ as above.

Finally, let $\phi_{0} \in C_{0}^{\infty}(\mathbb{R})$ be a cutoff with support in $[1 / 2,2]$, while $\phi(t)$ is smooth with $\phi=0$ for $t<1 / 2$ and $\phi=1$ for $t>1$; multiplying both sides of (A.10) by $\phi_{0}(\sqrt{x}) \phi(t)$ we get

$$
\phi_{0}(\sqrt{x}) \phi(t) e^{i t \sqrt{x}}=\rho(x t, t)+\phi_{0}(\sqrt{x}) \sqrt{t} \int_{0}^{\infty} e^{i t / 4 s} e^{i p s} a_{0}(s, t) d s
$$

where

$$
\rho(p, t)=\phi_{0}(\sqrt{p / t}) \sigma(p, t) \phi(t) \sqrt{t}, \quad a_{0}(s, t)=a(s) \phi(t) .
$$

It is easy to check that $\rho$ and $a_{0}$ satisfy the properties in the statement, using (A.8) and noticing the restriction $1 / 4 \leq p / t \leq 4$ imposed by $\phi_{0}$. A rescaling

$$
(x t, t) \rightarrow\left(\frac{x t}{\lambda}, \lambda t\right)
$$

concludes the proof.

\section{REFERENCES}

[1] Richard Beals. $L^{p}$ boundedness of Fourier integral operators. Mem. Amer. Math. Soc., 38(264):viii+57, 1982.

[2] Jacek Dziubański. Triebel-Lizorkin spaces associated with Laguerre and Hermite expansions. Proc. Amer. Math. Soc., 125(12):3547-3554, 1997.

[3] Jay Epperson. Triebel-Lizorkin spaces for Hermite expansions. Studia Math., 114(1):87-103, 1995.

[4] Arthur Erdélyi, Wilhelm Magnus, Fritz Oberhettinger, and Francesco G. Tricomi. Higher transcendental functions. Vols. I, II. McGraw-Hill Book Company, Inc., New York-TorontoLondon, 1953. Based, in part, on notes left by Harry Bateman.

[5] Jean Ginibre and Giorgio Velo. The global Cauchy problem for the nonlinear Schrödinger equation revisited. Ann. Inst. H. Poincaré Anal. Non Linéaire, 2(4):309-327, 1985.

[6] Godfrey H. Hardy and Marcel Riesz. The general theory of Dirichlet series. Cambridge University Press, London, 1915.

[7] Markus Keel and Terence Tao. Endpoint Strichartz estimates. Amer. J. Math., 120(5):955980, 1998

[8] Herbert Koch and Fulvio Ricci. Spectral projections for the twisted Laplacian. Studia Math., 180(2):103-110, 2007.

[9] Herbert Koch and Daniel Tataru. $L^{p}$ eigenfunction bounds for the Hermite operator. Duke Math. J., 128(2):369-392, 2005.

[10] Akihiko Miyachi. On some estimates for the wave equation in $L^{p}$ and $H^{p}$. J. Fac. Sci. Univ. Tokyo Sect. IA Math., 27(2):331-354, 1980.

[11] Detlef Müller and Fulvio Ricci. Analysis of second order differential operators on Heisenberg groups. I. Invent. Math., 101(3):545-582, 1990

[12] Detlef Müller and Andreas Seeger. Sharp $l^{p}$-estimates for the wave equation on heisenberg type groups. in preparation, 2008.

[13] Detlef Müller and Elias M. Stein. $L^{p}$-estimates for the wave equation on the Heisenberg group. Rev. Mat. Iberoamericana, 15(2):297-334, 1999.

[14] Gestur Ólafsson and Shijun Zheng. Function spaces associated with Schrödinger operators: the Pöschl-Teller potential. J. Fourier Anal. Appl., 12(6):653-674, 2006.

[15] Juan C. Peral. $L^{p}$ estimates for the wave equation. J. Funct. Anal., 36(1):114-145, 1980.

[16] Andreas Seeger, Christopher D. Sogge, and Elias M. Stein. Regularity properties of Fourier integral operators. Ann. of Math. (2), 134(2):231-251, 1991.

[17] Jalal Shatah and Michael Struwe. Geometric wave equations, volume 2 of Courant Lecture Notes in Mathematics. New York University Courant Institute of Mathematical Sciences, New York, 1998.

[18] Sundaram Thangavelu. Riesz transforms and the wave equation for the Hermite operator. Comm. Partial Differential Equations, 15(8):1199-1215, 1990.

[19] Sundaram Thangavelu. Harmonic analysis on the Heisenberg group, volume 159 of Progress in Mathematics. Birkhäuser Boston Inc., Boston, MA, 1998.

[20] Jingzhi Tie and M. W. Wong. Wave kernels of the twisted Laplacian. In Modern trends in pseudo-differential operators, volume 172 of Oper. Theory Adv. Appl., pages 107-115. Birkhäuser, Basel, 2007. 
[21] Kenji Yajima. Schrödinger evolution equations with magnetic fields. J. Analyse Math., 56:2976, 1991.

Piero D'Ancona: Unversità di Roma "La Sapienza", Dipartimento di Matematica, Piazzale A. Moro 2, I-00185 Roma, Italy

E-mail address: dancona@mat.uniroma1.it

Vittoria Pierfelice: Unversité d’Orléans, Bâtiment de matématiques - Route de Chartres B.P. 6759 - 45067 Orléans Cedex 2, France

E-mail address: Vittoria.Pierfelice@univ-orleans.fr

Fulvio Ricci: Scuola Normale Superiore, Classe di Scienze, Piazza dei Cavalieri, I-56100 Pisa, ItALy

E-mail address: f.ricci@sns.it 